\title{
A Mixed Traffic Assignment Problem and Its Upper Bound of Efficiency Loss
}

\author{
Xiao-Jun Yu ${ }^{1}$ and Jiancheng Long ${ }^{2}$ \\ ${ }^{1}$ School of Mathematics and Statistics, Guizhou University of Finance and Economics, Guiyang 550025, China \\ ${ }^{2}$ School of Transportation Engineering, Hefei University of Technology, Hefei 230009, China \\ Correspondence should be addressed to Jiancheng Long; jianchenglong@hfut.edu.cn
}

Received 23 February 2014; Accepted 29 April 2014; Published 15 May 2014

Academic Editor: X. Zhang

Copyright (C) 2014 X.-J. Yu and J. Long. This is an open access article distributed under the Creative Commons Attribution License, which permits unrestricted use, distribution, and reproduction in any medium, provided the original work is properly cited.

\begin{abstract}
A variational inequality (VI) formulation of a mixed traffic assignment problem associated with user equilibrium (UE) player and altruistic players (UE-AP) is developed. The users governed by the UE player use the paths with minimum actual travel cost, while the users governed by altruistic players use the paths with minimum perceived travel cost, which is a linear combination of the actual and marginal travel costs. A nonlinear programming method is adopted to derive the efficiency loss of the UE-AP mixed equilibrium with polynomial cost function. Finally, numerical examples are given to verify our analytical results.
\end{abstract}

\section{Introduction}

Traditionally, traffic assignment problems assume that travelers follow Wardrop's first and second principles or their extensions. Wardrop's first principle or the user equilibrium (UE) principle assumes that all users are identical, noncooperative, and rational in selecting the shortest route and know the exact travel cost they will encounter. At equilibrium, no one can reduce her/his travel cost by unilaterally choosing alternative route of the same origin-destination (OD) pair. Wardrop's second principle or the system optimum (SO) principle assumes that each user chooses her/his route cooperatively with other users to minimize the total system travel cost.

Most of traffic assignment problems addressed in literatures are with homogeneous users. However, it has long been recognized that users in a traffic network are heterogeneous in general. For example, Haurie and Marcotte [1] considered that there exist some noncooperative Cournot-Nash $(\mathrm{CN})$ players in the network and assumed that users belonging to the same player are fully cooperative but are fully competitive for users belonging to different players. Harker [2] developed a network model with consideration of mixed behaviors and assumed that distinct $\mathrm{CN}$ players control certain portions of users, and the other users follow the UE principle. The UE principle and SO principle were also combined in a traffic assignment problem to investigate the route guidance problems [3-5]. Yang et al. [6] formulated a mixed behavior network equilibrium model that simultaneously describes the routing behaviors of $\mathrm{UE}, \mathrm{SO}$, and $\mathrm{CN}$ players. Bhavathrathan and Mallikarjuna [7] explored the heterogeneous traffic in Indian road traffic and introduced an idea of diminishing density in macroscopic model.

The efficiency loss of traffic assignment problem was initially addressed by Roughgarden and Tardos [8]. The efficiency loss or price of anarchy is the largest ratio of the total system travel cost at the UE state to that at the SO state for given traffic demand. Whereafter, the efficiency loss of many other traffic assignment problems with homogeneous users was further investigated [9-14]. Roughgarden [15] examined the price of anarchy with consideration of heterogeneous players. Liu et al. [16] investigated the efficiency loss of mixed travel behavior in networks with advanced travel information systems (ATIS). Han and Yang [17] derived the efficiency loss caused by the multiclass and multicriterion traffic equilibrium. Yang et al. [18] investigated the efficiency loss of the selfish routing with the atomic $\mathrm{CN}$ players. Yu et al. [19] studied the efficiency loss caused by the multiclass stochastic traffic equilibrium assignment with fixed demand. Yu and Huang [20] investigated the upper bound of the efficiency loss of UE$\mathrm{CN}$ mixed equilibrium with polynomial cost functions. 
Most of existing works assume that each player is entirely selfish and aims to find the minimum travel cost route for herself/himself without regard to the external cost in the congested networks [8-20]. Experiments have shown that participants do not act absolutely selfishly even for simple games in controlled environments [21, 22]. Instead, their behavior can be either altruistic or malicious. Chen and Kempe [23] studied the efficiency loss of network with altruistic players. Karakostas et al. [24] considered the price of anarchy of selfish players and oblivious players, in which the oblivious users ignore congestion altogether and decide their routing on the basis of cheapest routes on a network without any flow whatsoever.

In this paper, we assume that there are a single UE player and several altruistic players (UE-AP) in the network. The UE player controls all the selfish users who follow UE principle in their routing decisions, while the altruistic players regard the external cost that they cause for other players in the network. The perceived travel cost of altruistic users governed by an altruistic player is a linear combination of the selfish component (i.e., the actual travel cost) and the altruistic component (i.e., the marginal travel cost). All the users belonging to the same altruistic player are assumed to have the same altruism coefficient. This paper develops a variational inequality (VI) formulation for the UE-AP mixed traffic assignment problem and adopts a nonlinear programming method to derive the efficiency loss of the mixed equilibrium with polynomial cost function.

The rest of this paper is organized as follows. In Section 2, the UE-AP mixed traffic assignment problem is proposed and formulated as a VI problem. In Section 3, a nonlinear programming approach is adopted to derive the upper bound of efficiency loss of the UE-AP mixed equilibrium with polynomial cost function. Numerical examples are provided in Section 4. Finally, Section 5 draws the conclusions.

\section{The UE-AP Mixed Traffic Assignment Problem}

In the UE-AP mixed traffic assignment problem, each player can control more than one OD pair, but, for notational purpose, each OD pair is supposed to be controlled by only one player. If several players compete over the same physical OD pair, the OD pair is copied and treated as a set of OD pairs. Thus, any ownership of the OD pairs can be represented $[2,6]$. The OD demands are assumed to be fixed. In a multidestination and strongly connected network $G, N$ denotes the set of nodes, whereas $A$ denotes the set of arcs (links). The following notations will be adopted throughout this paper:

\section{$U$ : the UE player in the network;}

$K$ : the set of altruistic players in the network;

$W^{U}$ : the set of OD pairs controlled by UE player;

$W^{k}$ : the set of OD pairs controlled by altruistic player $k \in K$;

$\beta_{k}$ : the altruism coefficient of altruistic player $k \in K$;
$W^{K}: W^{K} \equiv \bigcup_{k \in K} W^{k}$

$W: W \equiv W^{U} \cup W^{K}$;

$d_{w}$ : the demand between OD pair $w \in W$;

$R_{w}$ : the set of paths connecting OD pair $w \in W$;

$f_{r w}$ : the flow on path $r \in R_{w}, w \in W$;

$\delta_{a r}^{w}: 0-1$ indicator variable; it equals 1 if path $r \in R_{w}$ traverses link $a \in A$, and 0 otherwise;

$v_{a}^{U}$ : the flow of link $a$ arising out of the OD pair flows from the set $W^{U}$;

$\mathbf{v}^{U}: \mathbf{v}^{U} \equiv\left(\ldots, v_{a-1}^{U}, v_{a}^{U}, v_{a+1}^{U}, \ldots\right)$;

$v_{a}^{k}$ : the flow of link $a$ arising out of the OD flows from the set $W^{k}, k \in K$;

$\mathbf{v}_{a}^{K}: \mathbf{v}_{a}^{K} \equiv\left(\ldots, v_{a}^{k-1}, v_{a}^{k}, v_{a}^{k+1}, \ldots\right) ;$

$\mathbf{v}^{k}: \mathbf{v}^{k} \equiv\left(\ldots, v_{a-1}^{k}, v_{a}^{k}, v_{a+1}^{k}, \ldots\right)$;

$\mathbf{v}^{K}: \mathbf{v}^{K} \equiv\left(\ldots, \mathbf{v}^{k-1}, \mathbf{v}^{k}, \mathbf{v}^{k+1}, \ldots\right)$;

$v_{a}^{K}: v_{a}^{K}=\sum_{k \in K} v_{a}^{k}$

$v_{a}: v_{a}=v_{a}^{U}+v_{a}^{K}$;

$\mathbf{v}_{a}: \mathbf{v}_{a} \equiv\left(v_{a}^{U}, \mathbf{v}_{a}^{K}\right) \equiv\left(v_{a}^{U}, v_{a}^{1}, \ldots, v_{a}^{k}, \ldots\right) ;$

$\mathbf{v}: \mathbf{v} \equiv\left(\mathbf{v}^{U}, \mathbf{v}^{K}\right)$;

$t_{a}\left(v_{a}\right)$ : the average travel cost of traversing link $a \in A$, which is separable in link flows and is assumed to be twice continuously differentiable, convex, and strictly increasing function.

The feasible sets of link flows by the UE player and the altruistic players can be defined as follows [6], respectively:

$$
\begin{aligned}
& \Omega^{U}=\left\{\mathbf{v}^{U} \mid v_{a}^{U}=\sum_{w \in W^{U}} \sum_{r \in R_{w}} f_{r w} \delta_{a r}^{w}, a \in A ;\right. \\
&\left.\sum_{r \in R_{w}} f_{r w}=d_{w} ; f_{r w} \geq 0, r \in R_{w}, w \in W^{U}\right\}, \\
& \Omega^{k}=\left\{\mathbf{v}^{k} \mid v_{a}^{k}=\sum_{w \in W^{k}} \sum_{r \in R_{w}} f_{r w} \delta_{a r}^{w}, a \in A ;\right. \\
&\left.\sum_{r \in R_{w}} f_{r w}=d_{w} ; f_{r w} \geq 0, r \in R_{w}, w \in W^{k}\right\}, \\
& k \in K .
\end{aligned}
$$

Based on the results of $[21,23]$, we propose the following definitions for altruistic users.

Definition 1 (perceived link cost). The perceived cost of link $a$ is a linear combination of the actual and marginal travel costs of the link, given as follows:

$$
\begin{aligned}
t_{a}^{\beta}(\mathbf{v}) & =(1-\beta) t_{a}\left(v_{a}\right)+\beta\left(v_{a} t_{a}\left(v_{a}\right)\right) \\
& =t_{a}\left(v_{a}\right)+\beta v_{a} t_{a}^{\prime}\left(v_{a}\right),
\end{aligned}
$$

where $\beta \in(0,1]$ is the altruistic coefficient. 
Definition 2 ( $\beta$ altruistic user). Each $\beta$ altruistic user chooses a path $r$ so as to minimize the following perceived route cost:

$$
t_{r}^{\beta}(\mathbf{v})=\sum_{a \in r} t_{a}^{\beta}(\mathbf{v})=\sum_{a \in r} t_{a}\left(v_{a}\right)+\beta \sum_{a \in r} v_{a} t_{a}^{\prime}\left(v_{a}\right) .
$$

The users governed by UE player aim to minimize their travel cost under the current routing decisions of the altruistic players, which is equivalent to solve the following optimization problem:

$$
\min _{\mathbf{v}^{U} \in \Omega^{U}} \sum_{a \in A} \int_{0}^{v_{a}^{U}} t_{a}\left(v_{a}^{K}+x\right) \mathrm{d} x
$$

where $v_{a}^{K}$ is fixed for the optimization problem (4). Since $t_{a}\left(v_{a}\right)$ is strictly increasing, the minimization problem (4) has a unique solution.

The users governed by altruistic player $k \in K$ aim to minimize their perceived travel cost under the current routing decisions of other players, which is equivalent to solve the following optimization problem:

$$
\min _{\mathbf{v}^{k} \in \Omega^{k}} \sum_{a \in A} \int_{0}^{v_{a}^{k}} t_{a}^{\beta_{k}}\left(v_{a}^{U}+v_{a}^{-k}+x\right) \mathrm{d} x,
$$

where $v_{a}^{-k}=\sum_{i \in K, i \neq k} v_{a}^{i}, v_{a}^{U}$ and $v_{a}^{-k}$ are fixed, and $v_{a}^{k}$ is the decision variable. The monotonicity of $t_{a}\left(v_{a}\right)$ ensures that the minimization problem (5) has a unique solution.

If a link flow pattern simultaneously satisfies the optimality conditions of the optimization problems (4) and (5), then it is referred to as a UE-AP mixed equilibrium solution. Given the fact that the feasible sets for each player are disjoint, it is well known that the combination of the optimization problems (4) and (5) can be formulated as a VI problem, given as follows [25].

Lemma 3. A vector $\overline{\mathbf{v}}=\left(\overline{\mathbf{v}}^{U}, \overline{\mathbf{v}}^{K}\right) \in \Omega$ is an optimal solution of the UE-AP mixed equilibrium of problems (4) and (5) if and only if the vector $\overline{\mathbf{v}}$ satisfies the following VI problem:

$$
\sum_{a \in A}\left[t_{a}\left(\bar{v}_{a}\right)\left(v_{a}^{U}-\bar{v}_{a}^{U}\right)+\sum_{k \in K} t_{a}^{\beta_{k}}\left(\bar{v}_{a}\right)\left(v_{a}^{k}-\bar{v}_{a}^{k}\right)\right] \geq 0, \quad \mathbf{v} \in \Omega,
$$

where $\bar{v}_{a}=\bar{v}_{a}^{U}+\bar{v}_{a}^{K}=\bar{v}_{a}^{U}+\sum_{k \in K} \bar{v}_{a}^{k}, t_{a}^{\beta_{k}}\left(\bar{v}_{a}\right)=t_{a}\left(\bar{v}_{a}\right)+$ $\beta_{k} \bar{v}_{a} t_{a}^{\prime}\left(\bar{v}_{a}\right)$, and $\Omega=\Omega^{U} \times \prod_{k \in K} \Omega^{k}$.

It is easy to know that both $\Omega^{U}$ and $\Omega^{k}$ are nonempty compact convex set, and hence the feasible solution set $\Omega$ of the VI problem (6) is a nonempty compact convex set. Since $t_{a}\left(v_{a}\right)$ is also twice continuously differentiable, the solution existence of the VI problem (6) can be guaranteed. The link costs perceived by the UE player and altruistic players on link $a \in A$ can be jointly defined as a vector, given as follows:

$$
\begin{array}{r}
\mathbf{c}_{a}\left(v_{a}^{U}, v_{a}^{K}\right)=\left(t_{a}\left(v_{a}\right), \ldots, t_{a}\left(v_{a}\right)+\beta_{k} v_{a} t_{a}^{\prime}\left(v_{a}\right), \ldots\right), \\
k \in K .
\end{array}
$$

If $\mathbf{c}_{a}\left(v_{a}^{U}, v_{a}^{K}\right)$ is strictly monotone, then the solution uniqueness of the VI problem (6) can be guaranteed [25].

Let $\overline{\mathbf{v}}=\left(\overline{\mathbf{v}}^{U}, \overline{\mathbf{v}}^{K}\right)$ and $\overline{\mathbf{v}}_{A}=\left(\bar{v}_{a}\right), a \in A$, be an optimal solution of the VI problem (6) and the vector of the aggregate link flow of the UE-AP mixed equilibrium, respectively. Then, the total system travel cost can be expressed as follows:

$$
T(\overline{\mathbf{v}})=\sum_{a \in A} t_{a}\left(\bar{v}_{a}\right) \bar{v}_{a}=\sum_{a \in A} t_{a}\left(\bar{v}_{a}\right) \bar{v}_{a}^{U}+\sum_{a \in A} \sum_{k \in K} t_{a}\left(\bar{v}_{a}\right) \bar{v}_{a}^{k} .
$$

Let $\mathbf{v}^{\text {so }}=\left(\mathbf{v}^{\text {so }, U}, \mathbf{v}^{\text {so }, K}\right)$ and $\mathbf{v}_{A}^{\text {so }}=\left(v_{a}^{\text {so }}\right), a \in A$, respectively, be the optimal solution and the aggregate link flow of the following optimization problem:

$$
\min _{\mathbf{v} \in \Omega} \sum_{a \in A} t_{a}\left(v_{a}\right) v_{a} .
$$

Since $t_{a}\left(v_{a}\right)$ is strictly increasing and convex, the optimization problem (9) has a unique solution in terms of aggregate link flows $\mathbf{v}_{A}^{\text {so }}$. Note that the link flows $v_{a}^{\text {so, } U}$ and $v_{a}^{\mathrm{so}, k}, k \in K, a \in A$, may not be unique, but $v_{a}^{\mathrm{so}}=v_{a}^{\mathrm{so}, U}+$ $\sum_{k \in K} v_{a}^{\mathrm{so}, k}$ is unique. Thus, the efficiency loss of the UE-AP mixed equilibrium can be defined as follows:

$$
\rho=\frac{T(\overline{\mathbf{v}})}{T\left(\mathbf{v}^{\mathrm{so}}\right)}=\frac{\sum_{a \in A} t_{a}\left(\bar{v}_{a}\right) \bar{v}_{a}}{\sum_{a \in A} t_{a}\left(v_{a}^{\mathrm{so}}\right) v_{a}^{\mathrm{so}}},
$$

where $T\left(\mathbf{v}^{\text {so }}\right)$ measures the minimum total system travel cost and $T(\overline{\mathbf{v}})$ is the total system travel cost at the UE-AP mixed equilibrium. Obviously, $\rho \geq 1$ holds. In the next section, we will derive the upper bound of the value of $\rho$.

\section{Bounding the Efficiency Loss of the UE-AP Mixed Equilibrium}

In this section, the following assumption is adopted for link travel cost functions.

Assumption 4. The link travel cost function is polynomial and has the following form:

$$
\begin{gathered}
t_{a}\left(v_{a}\right)=t_{a}(0)+\alpha_{a}\left(v_{a}\right)^{p} \\
a \in A, \quad t_{a}(0) \geq 0, \quad \alpha_{a} \geq 0, \quad p \geq 1 .
\end{gathered}
$$

Let $\mathbf{v}^{\text {so }}=\left(\mathbf{v}^{\text {so }, U}, \mathbf{v}^{\text {so, } K}\right)$ be an optimal solution of the optimization problem (9). Note that $\mathbf{v}^{\mathrm{so}, U} \in \Omega^{U}, \mathbf{v}^{\mathrm{so}, k} \in \Omega^{k}$, $k \in K$. Substituting $\mathbf{v}=\mathbf{v}^{\text {so }}$ into the VI problem (6), we have

$$
\sum_{a \in A}\left[t_{a}\left(\bar{v}_{a}\right)\left(v_{a}^{\mathrm{so}, U}-\bar{v}_{a}^{U}\right)+\sum_{k \in K} t_{a}^{\beta_{k}}\left(\bar{v}_{a}\right)\left(v_{a}^{\mathrm{so}, k}-\bar{v}_{a}^{k}\right)\right] \geq 0 .
$$

Rearranging (12), we have

$$
\begin{aligned}
\sum_{a \in A} t_{a}\left(\bar{v}_{a}\right) \bar{v}_{a}^{U}+\sum_{a \in A} \sum_{k \in K} t_{a}\left(\bar{v}_{a}\right) \bar{v}_{a}^{k} \\
\leq \sum_{a \in A} t_{a}\left(\bar{v}_{a}\right) v_{a}^{\mathrm{so}, U}+\sum_{a \in A} \sum_{k \in K} t_{a}\left(\bar{v}_{a}\right) v_{a}^{\mathrm{so}, k} \\
\quad+\sum_{a \in A} \sum_{k \in K} \beta_{k} \bar{v}_{a} t_{a}^{\prime}\left(\bar{v}_{a}\right)\left(v_{a}^{\mathrm{so}, k}-\bar{v}_{a}^{k}\right) .
\end{aligned}
$$


Thus,

$$
\begin{aligned}
T(\overline{\mathbf{v}}) \leq & T\left(\mathbf{v}^{\mathrm{so}}\right)+\sum_{a \in A} v_{a}^{\mathrm{so}}\left[t_{a}\left(\bar{v}_{a}\right)-t_{a}\left(v_{a}^{\mathrm{so}}\right)\right] \\
& +\sum_{a \in A} \sum_{k \in K} \beta_{k} \bar{v}_{a} t_{a}^{\prime}\left(\bar{v}_{a}\right)\left(v_{a}^{\mathrm{so}, k}-\bar{v}_{a}^{k}\right) \\
\leq & T\left(\mathbf{v}^{\mathrm{so}}\right)+\max _{\mathbf{v} \in \Omega}\left\{\sum_{a \in A} v_{a}^{\mathrm{so}}\left[t_{a}\left(\bar{v}_{a}\right)-t_{a}\left(v_{a}^{\mathrm{so}}\right)\right]\right. \\
& \left.+\sum_{a \in A} \sum_{k \in K} \beta_{k} \bar{v}_{a} t_{a}^{\prime}\left(\bar{v}_{a}\right)\left(v_{a}^{\mathrm{so}, k}-\bar{v}_{a}^{k}\right)\right\} \\
\leq & T\left(\mathbf{v}^{\mathrm{so}}\right)+\max _{\mathbf{v} \geq 0}\left\{\begin{array}{c}
\sum_{a \in A} v_{a}^{\mathrm{so}}\left[t_{a}\left(\bar{v}_{a}\right)-t_{a}\left(v_{a}^{\mathrm{so}}\right)\right] \\
\left.+\sum_{a \in A} \sum_{k \in K} \beta_{k} \bar{v}_{a} t_{a}^{\prime}\left(\bar{v}_{a}\right)\left(v_{a}^{\mathrm{so}, k}-\bar{v}_{a}^{k}\right)\right\} .
\end{array}\right.
\end{aligned}
$$

The last inequality of (14) is satisfied because the set $\{\mathbf{v} \mid$ $\mathbf{v} \geq 0$ \} includes the feasible solution set $\Omega$.

Substituting (11) into (14), we have

$$
\begin{aligned}
T(\overline{\mathbf{v}}) \leq T\left(\mathbf{v}^{\mathrm{so}}\right)+\max _{\mathbf{v} \geq 0}\left\{\sum_{a \in A} \alpha_{a}\left[\left(\bar{v}_{a}\right)^{p}-\left(v_{a}^{\mathrm{so}}\right)^{p}\right] v_{a}^{\mathrm{so}}\right. \\
\\
\left.\quad+\sum_{a \in A} \alpha_{a} p\left(\bar{v}_{a}\right)^{p} \sum_{k \in K} \beta_{k}\left(v_{a}^{\mathrm{so}, k}-\bar{v}_{a}^{k}\right)\right\} .
\end{aligned}
$$

The following nonlinear programming is developed to get the upper bound of the second term on the right-hand side of (15):

$$
\max _{\mathbf{v}_{a} \geq 0}\left\{\alpha_{a}\left[\left(\bar{v}_{a}\right)^{p}-\left(v_{a}\right)^{p}\right] v_{a}+\alpha_{a} p\left(\bar{v}_{a}\right)^{p} \sum_{k \in K} \beta_{k}\left(v_{a}^{k}-\bar{v}_{a}^{k}\right)\right\} .
$$

Define a function $F\left(\mathbf{v}_{a}\right)=\alpha_{a}\left[\left(\bar{v}_{a}\right)^{p}-\left(v_{a}\right)^{p}\right] v_{a}+$ $\alpha_{a} p\left(\bar{v}_{a}\right)^{p} \sum_{k \in K} \beta_{k}\left(v_{a}^{k}-\bar{v}_{a}^{k}\right)$. Since the Hessian matrix of $F\left(\mathbf{v}_{a}\right)$ is negative semidefinite, $F\left(\mathbf{v}_{a}\right)$ is a concave function when $\mathbf{v}_{a} \geq 0$ and $p \geq 1$. Hence, any local optimal solutions of the optimization problem (16) guarantee the global maximum objective. Let $\lambda_{a}^{U}$ and $\lambda_{a}^{k}$ be the Lagrange multiplies associated with $v_{a}^{U} \geq 0$ and $v_{a}^{k} \geq 0, k \in K, a \in A$, respectively. Then, we can get the first-order optimality conditions of the optimization problem (16) as follows:

$$
\begin{array}{r}
\alpha_{a}\left(\bar{v}_{a}\right)^{p}-\alpha_{a}(p+1)\left(v_{a}\right)^{p}+\lambda_{a}^{U}=0, \quad \forall a \in A, \\
\lambda_{a}^{U} \geq 0, \quad \lambda_{a}^{U} v_{a}^{U}=0, \quad \forall a \in A, \\
\alpha_{a}\left(\bar{v}_{a}\right)^{p}-\alpha_{a}(p+1)\left(v_{a}\right)^{p}+\alpha_{a} p \beta_{k}\left(\bar{v}_{a}\right)^{p}+\lambda_{a}^{k}=0, \\
\forall a \in A, \quad k \in K, \\
\lambda_{a}^{k} \geq 0, \quad \lambda_{a}^{k} v_{a}^{k}=0, \quad \forall a \in A, \quad k \in K .
\end{array}
$$

Combining (17) with (18), we have $v_{a} \geq(1 /(1+p))^{1 / p} \bar{v}_{a}$. For each given link, we can find that the optimization problem (16) can reach its maximum if one and only one of the following two conditions is satisfied:

$$
\begin{aligned}
& v_{a}=\left(\frac{1}{1+p}\right)^{1 / p} \bar{v}_{a}, \\
& v_{a}>\left(\frac{1}{1+p}\right)^{1 / p} \bar{v}_{a} .
\end{aligned}
$$

If condition (21) is satisfied, (17) and (18) imply $\lambda_{a}^{U}=0$ and $v_{a}^{U} \geq 0$. Substituting (21) into (19), we have

$$
\alpha_{a} p \beta_{k}\left(\bar{v}_{a}\right)^{p}+\lambda_{a}^{k}=0, \quad k \in K
$$

Since all parameters in (23) are nonnegative, we have $\lambda_{a}^{k}=$ $0, k \in K$, and at least one of the three parameters $\bar{v}_{a}, \alpha_{a}$, and $\beta_{k}$ should be zero. If $\bar{v}_{a}=0$, then (21) implies $v_{a}=0$, and hence $F\left(\mathbf{v}_{a}\right)=0$. If $\alpha_{a}=0$, then the travel cost is a constant, and we also have $F\left(\mathbf{v}_{a}\right)=0$. If $\beta_{k}=0$, according to (16), we have

$$
\begin{aligned}
& \max _{\mathbf{v}_{a} \geq 0}\left\{\alpha_{a}\left[\left(\bar{v}_{a}\right)^{p}-\left(v_{a}\right)^{p}\right] v_{a}+\alpha_{a} p\left(\bar{v}_{a}\right)^{p} \sum_{k \in K} \beta_{k}\left(v_{a}^{k}-\bar{v}_{a}^{k}\right)\right\} \\
& =\alpha_{a}\left\{\left(\bar{v}_{a}\right)^{p}-\left[\left(\frac{1}{1+p}\right)^{1 / p} \bar{v}_{a}\right]^{p}\right\}\left(\frac{1}{1+p}\right)^{1 / p} \bar{v}_{a} \\
& =\alpha_{a}\left(\frac{p}{1+p}\right)\left(\frac{1}{1+p}\right)^{1 / p}\left(\bar{v}_{a}\right)^{p+1} \\
& \leq\left(\frac{p}{1+p}\right)\left(\frac{1}{1+p}\right)^{1 / p} t_{a}\left(\bar{v}_{a}\right) \bar{v}_{a} .
\end{aligned}
$$

The last inequality of (24) follows the fact that $t_{a}(0) \geq 0$, for all $a \in A$.

If (22) holds, referring to (17) and (18), we have $\lambda_{a}^{U}>0$ and $v_{a}^{U}=0$. Hence, (19) implies that $\lambda_{a}^{k_{1}}<\lambda_{a}^{k_{2}}$ if $\beta_{k_{1}}>\beta_{k_{2}}$. According to (20), we further have $v_{a}^{k_{1}} \geq v_{a}^{k_{2}}=0$. Let $\beta_{\bar{k}}$ and $\beta_{\tilde{k}}$ be the maximum and minimum altruism coefficient of altruistic player that has positive flow on link $a$, respectively. Since $v_{a}^{U}=0$ and $v_{a}=v_{a}^{U}+\sum_{k \in K} v_{a}^{k}$, (19) implies $v_{a}=v_{a}^{\bar{k}}=$ $\left(\left(1+p \beta_{\bar{k}}\right) /(1+p)\right)^{1 / p} \bar{v}_{a}$. Therefore, we have

$$
\begin{aligned}
\max _{\mathbf{v}_{a} \geq 0} & \left\{\alpha_{a}\left[\left(\bar{v}_{a}\right)^{p}-\left(v_{a}\right)^{p}\right] v_{a}+\alpha_{a} p\left(\bar{v}_{a}\right)^{p} \sum_{k \in K} \beta_{k}\left(v_{a}^{k}-\bar{v}_{a}^{k}\right)\right\} \\
= & \alpha_{a}\left(1-\beta_{\bar{k}}\right)\left(\frac{p}{1+p}\right)\left(\frac{1+p \beta_{\bar{k}}}{1+p}\right)^{1 / p}\left(\bar{v}_{a}\right)^{p+1} \\
& +\alpha_{a} p \beta_{\bar{k}}\left[\left(\frac{1+p \beta_{\bar{k}}}{1+p}\right)^{1 / p}-\gamma_{a}\right]\left(\bar{v}_{a}\right)^{p+1} \\
& -\alpha_{a} p\left(\bar{v}_{a}\right)^{p} \sum_{k \in K, k \neq \bar{k}} \beta_{k} \bar{v}_{a}^{k}
\end{aligned}
$$




$$
\begin{aligned}
\leq & \alpha_{a}\left(1-\beta_{\bar{k}}\right)\left(\frac{p}{1+p}\right)\left(\frac{1+p \beta_{\bar{k}}}{1+p}\right)^{1 / p}\left(\bar{v}_{a}\right)^{p+1} \\
& +\alpha_{a} p \beta_{\bar{k}}\left[\left(\frac{1+p \beta_{\bar{k}}}{1+p}\right)^{1 / p}-\gamma_{a}\right]\left(\bar{v}_{a}\right)^{p+1} \\
& -\alpha_{a} p \beta_{\bar{k}}\left(\bar{v}_{a}\right)^{p} \sum_{k \in K, k \neq \bar{k}} \bar{v}_{a}^{k} \\
= & \alpha_{a}\left(1-\beta_{\bar{k}}\right)\left(\frac{p}{1+p}\right)\left(\frac{1+p \beta_{\bar{k}}}{1+p}\right)^{1 / p}\left(\bar{v}_{a}\right)^{p+1} \\
& +\alpha_{a} p \beta_{\bar{k}}\left[\left(\frac{1+p \beta_{\bar{k}}}{1+p}\right)^{1 / p}-\gamma_{a}\right]\left(\bar{v}_{a}\right)^{p+1} \\
& -\alpha_{a} p \beta_{\bar{k}}\left(1-\gamma_{a}-\kappa_{a}\right)\left(\bar{v}_{a}\right)^{p+1} \\
= & \alpha_{a} \varsigma_{a}\left(\bar{v}_{a}\right)^{p+1} \leq \varsigma_{a} t_{a}\left(\bar{v}_{a}\right) \bar{v}_{a},
\end{aligned}
$$

where $\gamma_{a}=\bar{v}_{a}^{\bar{k}} / \bar{v}_{a}, \kappa_{a}=\bar{v}_{a}^{U} / \bar{v}_{a}$ for $a \in A$ with $0 \leq \gamma_{a} \leq 1$, $0 \leq \kappa_{a} \leq 1,0 \leq \gamma_{a}+\kappa_{a} \leq 1$, and

$$
\begin{aligned}
\varsigma_{a}= & \left(1-\beta_{\bar{k}}\right)\left(\frac{p}{1+p}\right)\left(\frac{1+p \beta_{\bar{k}}}{1+p}\right)^{1 / p} \\
& +p \beta_{\bar{k}}\left[\left(\frac{1+p \beta_{\bar{k}}}{1+p}\right)^{1 / p}-\gamma_{a}\right]-p \beta_{\widetilde{k}}\left(1-\gamma_{a}-\kappa_{a}\right) .
\end{aligned}
$$

The last inequality of (25) follows the fact that $t_{a}(0) \geq 0$, $a \in A$. If $\bar{v}_{a}=0$, then $v_{a}=0$ and $F\left(\mathbf{v}_{a}\right)=0$. In this case, we have $\varsigma_{a}=0$.

Then, (24) or (25) is satisfied when the objective of the optimization problem (16) achieved its maximum value. Let

$$
\begin{gathered}
\xi_{a}=\max \left\{\left(\frac{p}{1+p}\right)\left(\frac{1}{1+p}\right)^{1 / p}, \varsigma_{a}\right\}, \\
\xi=\max _{a \in A} \xi_{a} .
\end{gathered}
$$

Then, (15) can be rewritten as follows:

$$
T(\overline{\mathbf{v}}) \leq T\left(\mathbf{v}^{\mathrm{so}}\right)+\xi T(\overline{\mathbf{v}}) .
$$

Based on the above analysis, we have the following theorem.

Theorem 5. Under Assumption 4, let $\overline{\mathbf{v}}=\left(\overline{\mathbf{v}}^{U}, \overline{\mathbf{v}}^{K}\right) \in \Omega$ be an optimal solution of the VI problem (6) and let $\mathbf{v}^{\text {so }}=$ $\left(\mathbf{v}^{\mathrm{so}, U}, \mathbf{v}^{\mathrm{so}, K}\right) \in \Omega$ be an optimal solution of the optimization problem (9). Then, the efficiency loss of the UE-AP mixed equilibrium satisfies $\rho \leq 1 /(1-\xi)$, where $\xi$ is defined by (26)(28).

If there is only UE player in the network, that is, all users in the network are selfish, then only (24) is satisfied when the optimization problem (16) reaches its maximum.

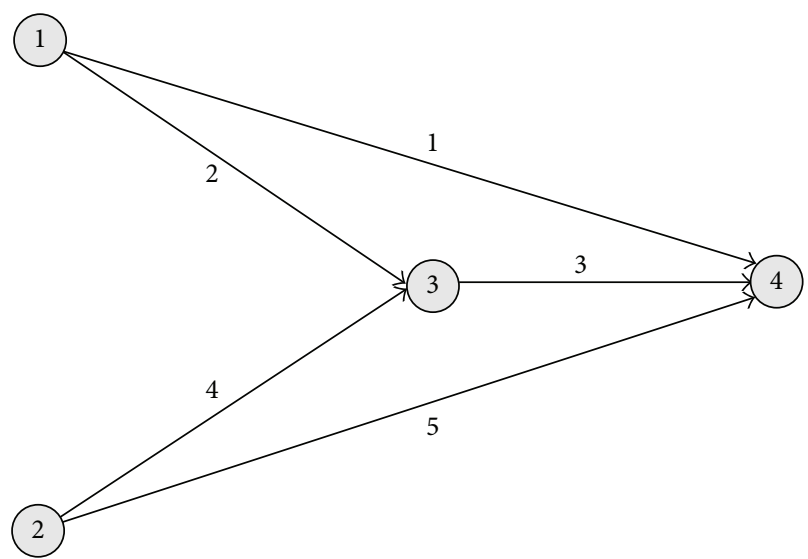

Figure 1: The network used in the example.

The efficiency loss of the equilibrium immediately follows $\rho \leq\left[1-(p /(1+p))(1 /(1+p))^{1 / p}\right]^{-1}$, which is consistent with the result in [12]. When the network only has altruistic players, then, only (25) is satisfied when the optimization problem (16) reaches its maximum. If $\kappa_{a}=0$ is satisfied for all $a \in A$, we can obtain that the efficiency loss of altruistic players is $\rho \leq 1 /(1-\psi)$, where $\psi=\max _{a \in A}\left\{\varsigma_{a}\right\}$, and

$$
\begin{aligned}
\varsigma_{a}= & \left(1-\beta_{\bar{k}}\right)\left(\frac{p}{1+p}\right)\left(\frac{1+p \beta_{\bar{k}}}{1+p}\right)^{1 / p} \\
& +p \beta_{\bar{k}}\left[\left(\frac{1+p \beta_{\bar{k}}}{1+p}\right)^{1 / p}-\gamma_{a}\right]-p \beta_{\widetilde{k}}\left(1-\gamma_{a}\right) .
\end{aligned}
$$

If $\beta_{k}=1$ for all $k \in K$, we have $\gamma_{a}=1$. This implies that $\varsigma_{a}=0$ and $\psi=0$. Then, $\rho=1$; that is, there are no efficiency loss if all players are perfectly altruistic.

\section{Numerical Example}

We developed a numerical example using the network presented in Figure 1, which consists of 4 nodes and 5 links. The link travel cost functions are $t_{1}=1.8, t_{2}=0.1 v_{2}, t_{3}=v_{3}$, $t_{4}=0.1 v_{4}$, and $t_{5}=2.7$. There are two OD pairs $(1,4)$ and $(2$, 4) with fixed demands $d_{14}=1$ and $d_{24}=1$, respectively.

We can obtain that the optimal SO link flow solution of the optimization problem (9) is $\mathbf{v}^{\text {so }}=[1,0,1,1,0]$ and the total travel cost is 2.9. The following two cases were examined.

Case A. OD pair $(1,4)$ is controlled by a UE player (denoted by " $U$ "), and OD pair $(2,4)$ is controlled by an altruism player (denoted by " $\beta$ "); the altruism coefficient $\beta=0.5$.

Case B. The two OD pairs are controlled by altruism player, but the altruism coefficient is different. The OD pair (1, 4 ) is controlled by altruistic player " $\beta_{1}$ " and the altruism coefficient $\beta_{1}=0.2$, and OD pair $(2,4)$ is controlled by altruistic player " $\beta_{2}$ " and the altruism coefficient $\beta_{2}=0.7$. 
For case A, the UE-AP mixed equilibrium solution is given as follows:

$$
\begin{gathered}
\bar{v}_{1}^{U}=0.1429, \quad \bar{v}_{2}^{U}=0.8571, \\
\bar{v}_{3}^{U}=0.8571, \quad \bar{v}_{4}^{U}=0, \quad \bar{v}_{5}^{U}=0, \\
\bar{v}_{1}^{\beta}=0, \quad \bar{v}_{2}^{\beta}=0, \quad \bar{v}_{3}^{\beta}=0.8571, \\
\bar{v}_{4}^{\beta}=0.8571, \quad \bar{v}_{5}^{\beta}=0.1429 .
\end{gathered}
$$

The aggregate link flow is $\bar{v}_{1}=0.1429, \bar{v}_{2}=0.8571, \bar{v}_{3}=$ $1.7142, \bar{v}_{4}=0.8571$, and $\bar{v}_{5}=0.1429$, which generates the system's total travel cost that is 3.7285 . Thus, the efficiency loss is 1.2857. Using (26)-(28), we can obtain $\xi=0.3125$ and the upper bound of efficiency loss $\rho \leq(1-\xi)^{-1}=1.4545$. This result is consistent with Theorem 5 .

For case B, the UE-AP mixed equilibrium solution is given as follows:

$$
\begin{gathered}
\bar{v}_{1}^{\beta_{1}}=0.5455, \quad \bar{v}_{2}^{\beta_{1}}=0.4545, \\
\bar{v}_{3}^{\beta_{1}}=0.4545, \quad \bar{v}_{4}^{\beta_{1}}=0, \quad \bar{v}_{5}^{\beta_{1}}=0, \\
\bar{v}_{1}^{\beta_{2}}=0, \quad \bar{v}_{2}^{\beta_{2}}=0, \\
\bar{v}_{3}^{\beta_{2}}=1, \quad \bar{v}_{4}^{\beta_{2}}=1, \quad \bar{v}_{5}^{\beta_{2}}=0 .
\end{gathered}
$$

The aggregate link flow is $\bar{v}_{1}=0.5455, \bar{v}_{2}=0.4545, \bar{v}_{3}=$ $1.4545, \bar{v}_{4}=1$, and $\bar{v}_{5}=0$, which generates the total system travel cost of 3.2181. Thus, the efficiency loss is 1.1097. Using (30), we have $\psi=0.1787$ and the upper bound of efficiency loss $\rho \leq(1-\psi)^{-1}=1.2176$. The result is also consistent with Theorem 5 .

\section{Conclusion}

In this paper, the UE-AP mixed traffic assignment problem was proposed and formulated as a VI problem. We assumed that there are two categories of players in the network: the UE player and altruistic players. The former only has the selfish users, each of the later controls the altruistic users who have the same altruism coefficient. The efficiency loss of the mixed traffic equilibrium under the polynomial cost function was analytically developed. We found that the upper bounds of the UE-AP are independent with network topology but depend on the degree of the link travel cost functions and the maximum and minimum altruism coefficient. The results also show that the efficiency losses in existing literatures are special cases of our model. In the future, we will investigate the efficiency loss of the UE-AP mixed equilibrium with elastic demand.

\section{Conflict of Interests}

The authors declare that they have no conflict of interests regarding the publication of this paper.

\section{Acknowledgments}

This research was partly funded by the National Natural Science Foundation of China $(71161005,71271075)$, the Nomarch Foundation of Guizhou Province (2011067), and the Program for New Century Excellent Talents in University (NCET-130766).

\section{References}

[1] A. Haurie and P. Marcotte, "On the relationship between NashCournot and Wardrop equilibria," Networks, vol. 15, no. 3, pp. 295-308, 1985.

[2] P. T. Harker, "Multiple equilibrium behaviors on networks," Transportation Science, vol. 22, no. 1, pp. 39-46, 1988.

[3] T. van Vuren, D. van Vliet, and M. J. Smith, "Combined equilibrium in a network with partial route guidance," in Traffic Control Methods, S. Yagar and S. E. Rowe, Eds., 1990.

[4] T. van Vuren and D. P. Watling, "A multiple user class assignment model for route guidance," Transportation Research Record, no. 1306, pp. 22-31, 1991.

[5] M. J. Maher and P. C. Hughes, "Estimation of the potential benefits from an ATT system using a multiple user class stochastic user equilibrium model," in Application of Advance Technologies in Transportation Engineering, Y. J. Stephanedes and F. Filippi, Eds., pp. 700-704, 1995.

[6] H. Yang, X. Zhang, and Q. Meng, "Stackelberg games and multiple equilibrium behaviors on networks," Transportation Research B: Methodological, vol. 41, no. 8, pp. 841-861, 2007.

[7] B. K. Bhavathrathan and C. Mallikarjuna, "Evolution of macroscopic models for modeling the heterogeneous traffic: an Indian perspective," Transportation Letters, vol. 4, no. 1, pp. 29-39, 2012.

[8] T. Roughgarden and É. Tardos, "How bad is selfish routing?" Journal of the ACM, vol. 49, no. 2, pp. 236-259, 2002.

[9] C. K. Chau and K. M. Sim, "The price of anarchy for nonatomic congestion games with symmetric cost maps and elastic demands," Operations Research Letters, vol. 31, no. 5, pp. 327334, 2003.

[10] J. R. Correa, A. S. Schulz, and N. E. Stier-Moses, "Selfish routing in capacitated networks," Mathematics of Operations Research, vol. 29, no. 4, pp. 961-976, 2004.

[11] J. R. Correa, A. S. Schulz, and N. E. Stier-Moses, "On the inefficiency of equilibria in congestion games," in Proceedings of the 11th International Conference on Integer Programming and Combinatorial Optimization (IPCO '05), pp. 167-181, June 2005.

[12] T. Roughgarden and É. Tardos, "Bounding the inefficiency of equilibria in nonatomic congestion games," Games and Economic Behavior, vol. 47, no. 2, pp. 389-403, 2004.

[13] H.-J. Huang, L.-Q. Ouyang, and T.-L. Liu, "Upper bounds of efficiency loss for user equilibrium behavior in traffic networks," Journal of Beijing University of Aeronautics and Astronautics, vol. 32, no. 10, pp. 1215-1219, 2006.

[14] X. Guo, H. Yang, and T.-L. Liu, "Bounding the inefficiency of logit-based stochastic user equilibrium," European Journal of Operational Research, vol. 201, no. 2, pp. 463-469, 2010.

[15] T. Roughgarden, "Stackelberg scheduling strategies," in Proceedings of the 33rd Annual ACM Symposium on Theory of Computing, pp. 104-113, July 2001.

[16] T.-L. Liu, L.-Q. Ouyang, and H.-J. Huang, "Mixed travel behavior in networks with ATIS and upper bound of efficiency 
loss," System Engineering: Theory \& Practice, vol. 27, no. 4, pp. 154-159, 2007.

[17] D. Han and H. Yang, "The multi-class, multi-criterion traffic equilibrium and the efficiency of congestion pricing," Transportation Research E: Logistics and Transportation Review, vol. 44, no. 5, pp. 753-773, 2008.

[18] H. Yang, D. Han, and H. K. Lo, "Efficiency of atomic splittable selfish routing with polynomial cost functions," Networks and Spatial Economics, vol. 8, no. 4, pp. 443-451, 2008.

[19] X.-J. Yu, H.-J. Huang, and T.-L. Liu, "Efficiency loss of the multi-class stochastic traffic equilibrium assignment with fixed demand," Journal of Transportation Systems Engineering and Information Technology, vol. 9, no. 4, pp. 83-89, 2009.

[20] X. Yu and H.-J. Huang, "Efficiency loss of mixed equilibrium behaviors with polynomial cost functions," Promet-Traffic \& Traffico, vol. 22, no. 5, pp. 325-331, 2010.

[21] J. Ledyard, "Public goods: a survey of experimental research," in Handbook of Experimental Economics, J. Kagel and A. Roth, Eds., 1997.

[22] D. K. Levine, "Modeling altruism and spitefulness in experiments," Review of Economic Dynamics, vol. 1, no. 3, pp. 593-622, 1998.

[23] P.-A. Chen and D. Kempe, "Altruism, selfishness, and spite in traffic routing," in Proceedings of the 9th ACM Conference on Electronic Commerce (EC '08), pp. 140-149, July 2008.

[24] G. Karakostas, T. Kim, A. Viglas, and H. Xia, "On the degradation of performance for traffic networks with oblivious users," Transportation Research B: Methodological, vol. 45, no. 2, pp. 364-371, 2011.

[25] D. Kinderlehrer and G. Stampacchia, An Introduction to Variational Inequalities and Their Applications, Academic Press, New York, NY, USA, 1986. 


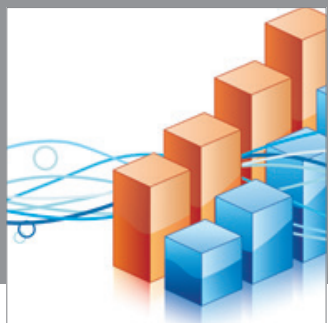

Advances in

Operations Research

mansans

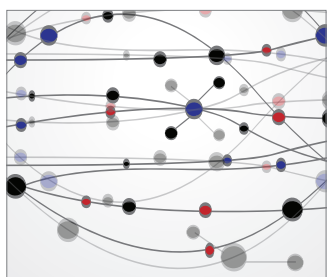

The Scientific World Journal
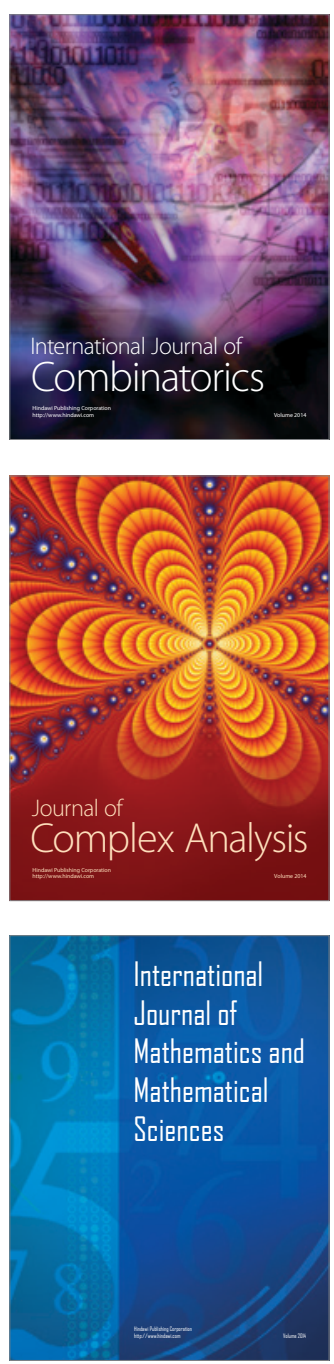
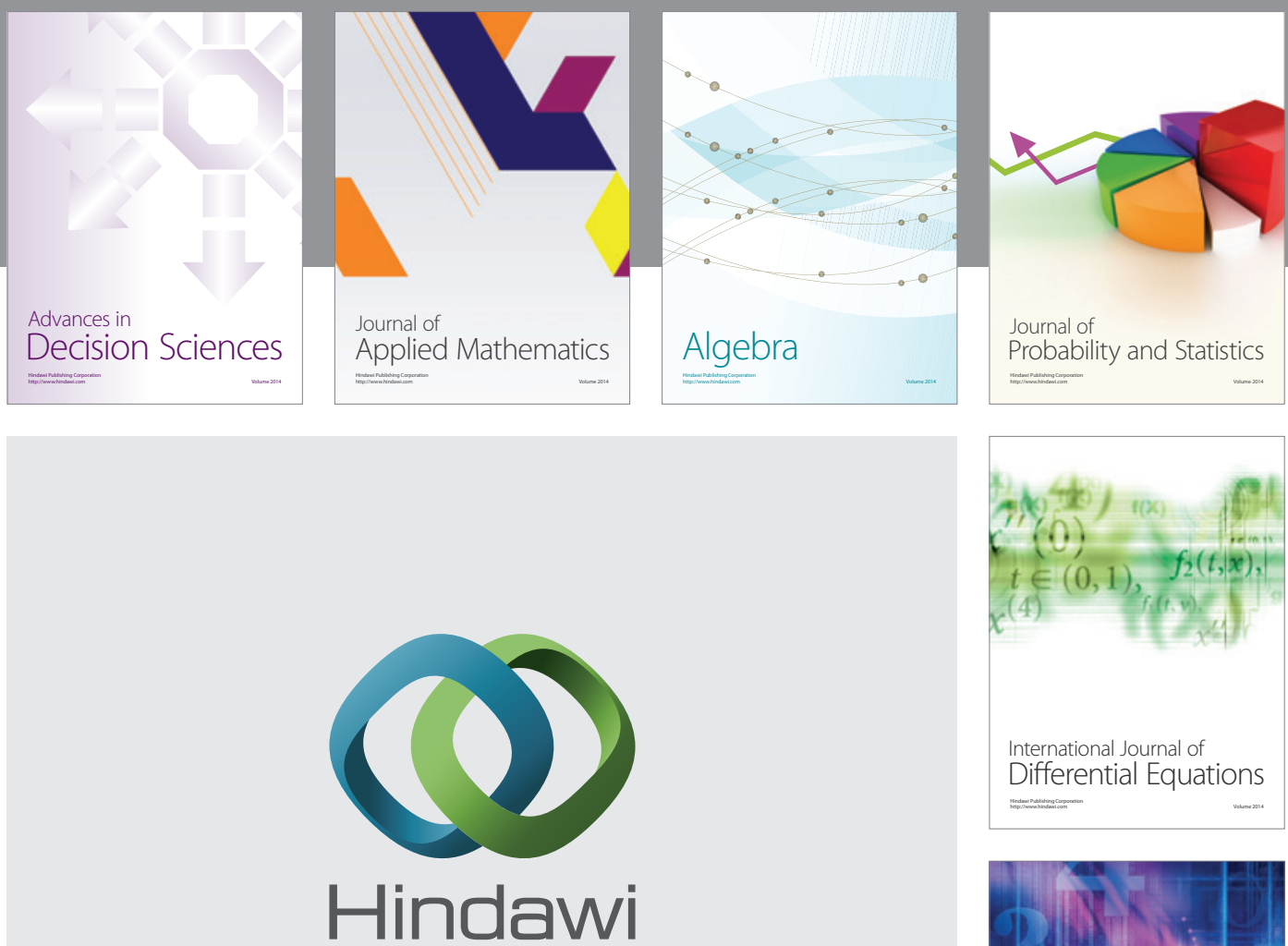

Submit your manuscripts at http://www.hindawi.com
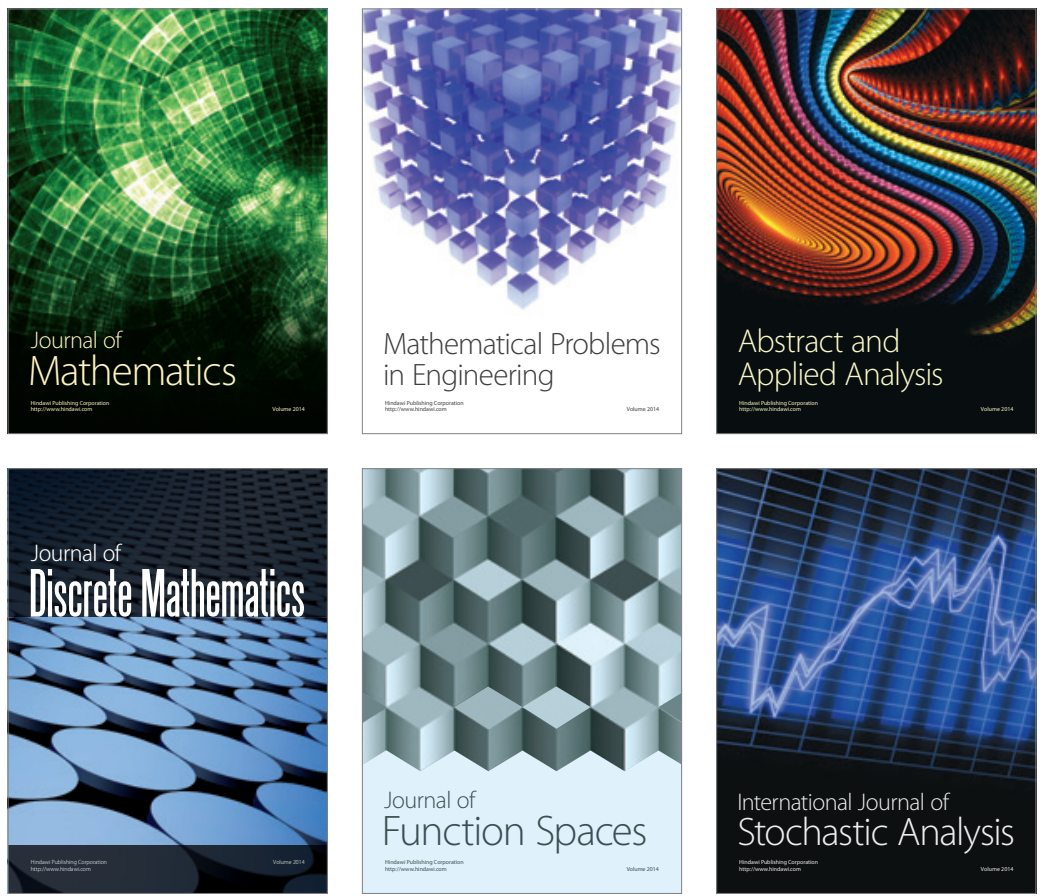

Journal of

Function Spaces

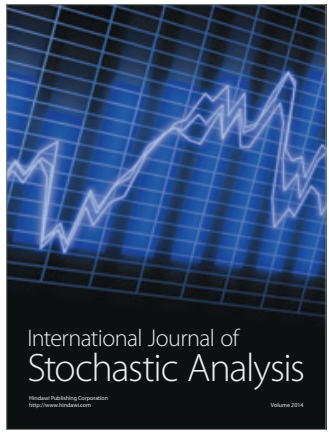

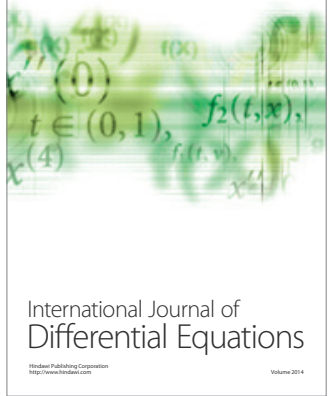
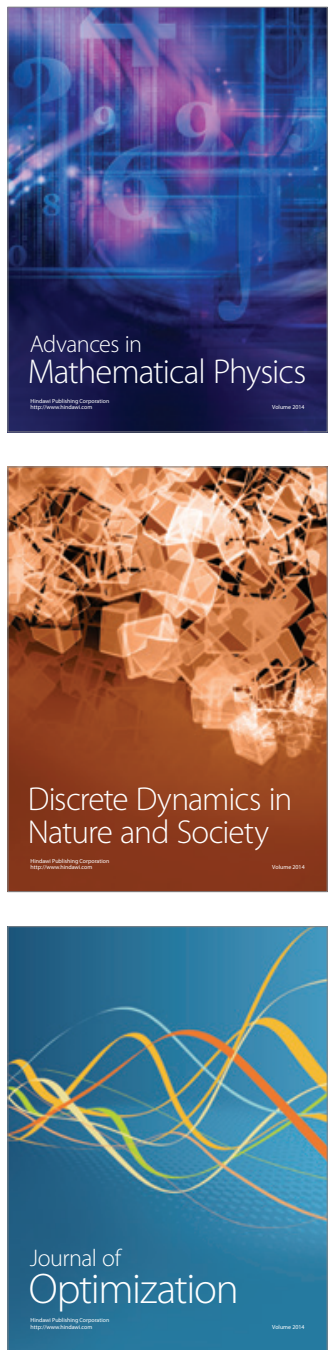